\title{
Magnetic $\mathrm{Fe}_{2} \mathrm{P}$ Nanowires and $\mathrm{Fe}_{2} \mathrm{P} @ \mathrm{C}$ Core@Shell Nanocables
}

\author{
Junli Wang ${ }^{1,2}$, Qing Yang ${ }^{1,2}(\bowtie)$, Jun Zhou ${ }^{1}$, Kewen Sun ${ }^{2}$, Zude Zhang ${ }^{2}$, Xiaoming Feng ${ }^{1}$, and Tanwei Li ${ }^{1}$ \\ ${ }^{1}$ Hefei National Laboratory for Physical Science at Microscale, University of Science and Technology of China, Hefei 230026, China \\ ${ }^{2}$ Department of Chemistry, University of Science and Technology of China, Hefei 230026, China \\ Received: 18 October 2009 / Revised: 21 January 2010 / Accepted: 22 January 2010 \\ (C) The Author(s) 2010. This article is published with open access at Springerlink.com
}

\begin{abstract}
We report the synthesis of one-dimensional (1-D) magnetic $\mathrm{Fe}_{2} \mathrm{P}$ nanowires and $\mathrm{Fe}_{2} \mathrm{P} @ \mathrm{C}$ core@shell nanocables by the reactions of triphenylphosphine $\left(\mathrm{PPh}_{3}\right)$ with Fe powder (particles) and ferrocene $\left(\mathrm{Fe}\left(\mathrm{C}_{5} \mathrm{H}_{5}\right)_{2}\right)$, respectively, in vacuum-sealed ampoules at $380-400{ }^{\circ} \mathrm{C}$. The synthesis is based on chemical conversion of micrometer or nanometer sized Fe particles into $\mathrm{Fe}_{2} \mathrm{P}$ via the extraction of phosphorus from liquid $\mathrm{PPh}_{3}$ at elevated temperatures. In order to control product diameters, a convenient sudden-temperature-rise strategy is employed, by means of which diameter-uniform $\mathrm{Fe}_{2} \mathrm{P} @ \mathrm{C}$ nanocables are prepared from the molecular precursor $\mathrm{Fe}\left(\mathrm{C}_{5} \mathrm{H}_{5}\right)_{2}$. In contrast, this strategy gives no obvious control over the diameters of the $\mathrm{Fe}_{2} \mathrm{P}$ nanowires obtained using elemental $\mathrm{Fe}$ as iron precursor. The formation of 1-D Fe $2 \mathrm{P}$ nanostructures is ascribed to the cooperative effects of the kinetically induced anisotropic growth and the intrinsically anisotropic nature of hexagonal $\mathrm{Fe}_{2} \mathrm{P}$ crystals. The resulting $\mathrm{Fe}_{2} \mathrm{P}$ nanowires and $\mathrm{Fe}_{2} \mathrm{P} @ \mathrm{C}$ nanocables display interesting ferromagnetic-paramagnetic transition behaviors with blocking temperatures of 230 and $268 \mathrm{~K}$, respectively, significantly higher than the ferromagnetic transition temperature of bulk $\mathrm{Fe}_{2} \mathrm{P}\left(T_{\mathrm{C}}=217 \mathrm{~K}\right)$.
\end{abstract}

\section{KEYWORDS}

Metal phosphide, nanowires, core@shell nanocables, magnetic nanostructures, chemical synthesis

\section{Introduction}

One-dimensional (1-D) nanostructures, including nanorods, nanowires, nanotubes, and nanocables, are attracting broad interest owing to the unique properties arising from their anisotropic shape and their promising applications in nanowire-based devices [1-5]. Transition metal phosphides are one significant type of functional material and exhibit a wide range of properties including ferromagnetism, superconductivity, magnetocaloric effect, magnetoresistance, catalysis, and lithium intercalation [6-10]. It is noted that, compared to the progress in the controllable synthesis of 1-D nanostructured metals, metal oxides and chalcogenides, and IV and III-V semiconductors [1, 2, 11-20], there have been fewer successful preparations of 1-D nanostructures of transition metal phosphides because of the difficulties in chemical synthesis and the lack of appropriate synthetic methodologies [21, 22]. In this respect, it is of much interest and fundamental importance to explore effective methods for preparing 1-D transition metal phosphide nanostructures.

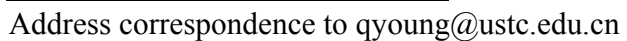


In recent years, several solution-mediated methods have been reported for the preparation of 1-D transition metal phosphide nanostructures. The solution-based precursor injection method was successfully utilized to synthesize high-quality nanowires/nanorods of transition metal phosphides such as $\mathrm{MnP}, \mathrm{FeP}, \mathrm{Fe}_{2} \mathrm{P}$, $\mathrm{Co}_{2} \mathrm{P}$, and $\mathrm{Ni}_{2} \mathrm{P}$ [22-26]. In these syntheses, continuous addition of precursor molecules into the reaction solution enabled anisotropic 1-D growth of the metal phosphide nanocrystals, and the diameter and aspect ratio of the nanowires or nanorods can be further tuned by controlling either the injection rate or the choice of surfactants [22-26]. Meanwhile, the O'Brien group reported an one-pot reflux route for the synthesis of CoP nanowires through thermal decomposition of [Co(acac $\left.)_{2}\right]($ acac $=$ acetylacetonate $)$ and tetradecylphosphonic acid in a mixed surfactant solution of trioctylphosphine oxide (TOPO) and hexadecylamine (HDA) at $340{ }^{\circ} \mathrm{C}$ for $3 \mathrm{~h}$ [27], in which the anisotropic 1-D growth of CoP nanowires was achieved by employing selective adsorption of surfactants to control the growth rates of different facets. Recently, Whitmire and co-workers showed that $\mathrm{Fe}_{2} \mathrm{P}$ nanorods and bundles can be prepared by the thermal decomposition of a single-source precursor $\left(\mathrm{H}_{2} \mathrm{Fe}_{3}(\mathrm{CO})_{9} \mathrm{P}^{\mathrm{t}} \mathrm{Bu}\right)$ in a mixture of trioctylamine (TOA) and oleic acid (OAc) at temperatures from 315 to $330{ }^{\circ} \mathrm{C}$ depending on the ratios of TOA and OA [28]. In these methods, a mixture of two or more surfactants was required, and the formation of 1-D transition metal phosphide nanostructures is usually explained by the cooperative effects of the surfactants, such as selective adsorption, along with the intrinsically anisotropic crystal structure of the phosphides [22-28]. Other methods, such as high-temperature thermal treatment of single-molecule precursors [29], hydrothermal and solvothermal methods [30, 31], the de-silylation strategy [21, 32], phosphate reduction [33], and metal particle conversion in solution [34-36], produced spherical nanoparticles of transition metal phosphides.

In our early work, we synthesized transition metal phosphide nanowires/nanorods by new Ullmann-type reactions between elemental transition metals and triphenylphosphine $\left(\mathrm{PPh}_{3}\right)$ [37], in which $\mathrm{Fe}_{2} \mathrm{P}$ nanowires were produced. However, the as-obtained $\mathrm{Fe}_{2} \mathrm{P}$ nanowires had broad size distribution, and their magnetic properties and anisotropic 1-D growth mechanism were not clarified. Recently, we replaced the elemental metal by a molecular metal precursor in the preparation of nanoscale transition metal phosphides and successfully obtained $\mathrm{Fe}_{2} \mathrm{P} @ \mathrm{C}$ core@shell nanocables [38]. Inspired by the precursor injection method [14, 15, 22-26], we herein propose a suddentemperature-rise strategy for controlling the product size and shape. The experimental results show that a sudden rise in reaction temperature (from 220 to $400{ }^{\circ} \mathrm{C}$ ) can effectively control the diameter distribution of $\mathrm{Fe}_{2} \mathrm{P} @ \mathrm{C}$ nanocables obtained using a molecular precursor (ferrocene, $\mathrm{Fe}\left(\mathrm{C}_{5} \mathrm{H}_{5}\right)_{2}$ ) as the iron source, but had no influence on the diameter control of $\mathrm{Fe}_{2} \mathrm{P}$ nanowires synthesized from elemental Fe powder. In this study, we investigate the reaction process, nucleation and growth mechanisms, and the effects of the iron precursors (in elemental or molecular states) and heating rate on the size and shape control of 1-D $\mathrm{Fe}_{2} \mathrm{P}$ nanostructures, by carefully analyzing the two syntheses of $\mathrm{Fe}_{2} \mathrm{P}$ nanowires and $\mathrm{Fe}_{2} \mathrm{P} @ \mathrm{C}$ core@shell nanocables. Moreover, the magnetic properties of the resulting $\mathrm{Fe}_{2} \mathrm{P}$ nanowires and $\mathrm{Fe}_{2} \mathrm{P} @ \mathrm{C}$ nanocables were compared and shown to exhibit different magnetic behavior from each other, and from bulk $\mathrm{Fe}_{2} \mathrm{P}$.

\section{Experimental}

\subsection{Synthesis of $\mathrm{Fe}_{2} \mathrm{P}$ nanowires}

All the reagents were purchased from Sinopharm Chemical Reagent Co. Ltd of Shanghai. Iron powder (Fe, 98\%) was first treated with $0.5 \mathrm{~mol} / \mathrm{L} \mathrm{NaBH}_{4}$ aqueous solution by sonication to remove surface oxides [37]. The synthesis of $\mathrm{Fe}_{2} \mathrm{P}$ nanowires described here was carried out in a metal-rich reaction and similar to that reported in our early work [37]. In detail, a mixture of $\mathrm{NaBH}_{4}$-treated Fe powder and $\mathrm{PPh}_{3}(99 \%)$ with a molar ratio of 4:1-3:1 was put into a quartz ampoule $(\phi 8 \mathrm{~mm} \times 150 \mathrm{~mm})$, and the ampoule was then evacuated and sealed. After that, the sealed ampoule was loaded into a resistance furnace with a tilt angle of $5^{\circ}$, heated from room temperature to $220^{\circ} \mathrm{C}$ in $30 \mathrm{~min}$, and kept at this temperature for $1 \mathrm{~h}$. The ampoule was then quickly transferred into a $380^{\circ} \mathrm{C}$ resistance furnace, and kept at this temperature for 
$15 \mathrm{~h}$ to prepare $\mathrm{Fe}_{2} \mathrm{P}$ nanowires. After the ampoule was naturally cooled to room temperature, the black products were collected, washed successively with benzene, dilute hydrochloric acid at $70{ }^{\circ} \mathrm{C}$ for several hours to remove unreacted $\mathrm{Fe}$, and absolute alcohol. The products were finally dried in a vacuum furnace at $60^{\circ} \mathrm{C}$.

\subsection{Synthesis of $\mathrm{Fe}_{2} \mathrm{P} @ \mathrm{C}$ core@shell nanocables}

The detailed process for the synthesis of $\mathrm{Fe}_{2} \mathrm{P} @ \mathrm{C}$ is similar to that in our previous work [38]. Briefly, a mixture of $2 \mathrm{mmol} \mathrm{Fe}\left(\mathrm{C}_{5} \mathrm{H}_{5}\right)_{2}(0.372 \mathrm{~g}$, 98\%) and 1.5 $\mathrm{mmol} \mathrm{PPh}_{3}(0.400 \mathrm{~g})$ was loaded into a quartz ampoule $(\phi 8 \mathrm{~mm} \times 150 \mathrm{~mm})$. Then, the ampoule was evacuated and sealed. The subsequent procedures were similar to what were used for the synthesis of $\mathrm{Fe}_{2} \mathrm{P}$ nanowires. The sudden rise in temperature was from 220 to $400{ }^{\circ} \mathrm{C}$ and the reaction was kept at $400{ }^{\circ} \mathrm{C}$ for $90 \mathrm{~min}$ in order to prepare $\mathrm{Fe}_{2} \mathrm{P} @ \mathrm{C}$ core@shell nanocables. It should be pointed out that, when heated at $220^{\circ} \mathrm{C}$, the reactant mixture became a red homogeneous liquid in the sealed ampoule.

\subsection{Characterization}

The crystal structure and phase purity of the $\mathrm{Fe}_{2} \mathrm{P}$ nanowires and $\mathrm{Fe}_{2} \mathrm{P} @ \mathrm{C}$ nanocables were examined by powder X-ray diffraction (XRD, Philips X'Pert Pro Super diffractometer) with graphite-monochromatized $\mathrm{Cu}$ K $\alpha$ radiation $(\lambda=1.54178 \AA$ ). Field emission scanning electron microscopy (FESEM) images were taken on a FEI Sirion-200 SEM. Electron diffraction (ED) patterns, energy dispersive X-ray (EDX) spectra, and lowresolution transmission electron microscope (TEM) and high-resolution (HRTEM) micrographs were collected on a JEOL JEM-2011 electron microscope operating at $200 \mathrm{kV}$. Samples for TEM analysis were prepared by sonicating the products in ethanol and dropping a small volume onto a carbon-coated copper grid. The magnetic properties of $\mathrm{Fe}_{2} \mathrm{P}$ nanowires and $\mathrm{Fe}_{2} \mathrm{P} @ \mathrm{C}$ nanocables were measured using a quantum design MPMS-XL7 superconducting quantum interference device (SQUID) magnetometer, and the temperature dependence of magnetization was measured in an applied magnetic field of $100 \mathrm{Oe}$ between 2 and $400 \mathrm{~K}$ using zero-field-cooling (ZFC) and field-cooling (FC) procedures.

\section{Results and discussion}

The synthesis of 1-D $\mathrm{Fe}_{2} \mathrm{P}$ nanowires and $\mathrm{Fe}_{2} \mathrm{P} @ \mathrm{C}$ coaxial core@shell nanocables was based on the chemical reactions of the iron precursors metallic iron and ferrocene $\left(\mathrm{Fe}\left(\mathrm{C}_{5} \mathrm{H}_{5}\right)_{2}\right)$ with $\mathrm{PPh}_{3}$ at temperatures of 380 and $400{ }^{\circ} \mathrm{C}$, respectively, as described in the Experimental section. Figure 1 shows the XRD patterns for the nanowires and nanocables obtained in typical synthetic procedures. All of the diffraction peaks in both patterns can be indexed to hexagonal structured $\mathrm{Fe}_{2} \mathrm{P}$ with the space group $P \overline{6} 2 \mathrm{~m}$, and are in good agreement with the reported data $(a=5.865 \AA$, and $c=3.456 \AA$, JCPDS 88-1803). The peaks correspond to the (111), (201), (210), (002), and (300) planes of $\mathrm{Fe}_{2} \mathrm{P}$. No extra peaks from impurities are observed. The overall morphologies of the products were examined with FESEM (Fig. 2). FESEM images show that all of the products have 1-D wire-like structures, and reveal that the $\mathrm{Fe}_{2} \mathrm{P}$ nanowires (Fig. 2(a)) have a wide diameter distribution ranging from 10 to $100 \mathrm{~nm}$, while the $\mathrm{Fe}_{2} \mathrm{P} @ \mathrm{C}$ nanocables (shown in Fig. 2(b)) possess an average diameter of $50 \mathrm{~nm}$ with a narrow diameter distribution. It is further notable that the nanocables are flexible and have a high aspect ratio.

The detailed morphologies of $\mathrm{Fe}_{2} \mathrm{P}$ nanowires and $\mathrm{Fe}_{2} \mathrm{P} @ \mathrm{C}$ core@shell nanocables were investigated by TEM. TEM images (Figs. 3(a) and 3(b)) show that the $\mathrm{Fe}_{2} \mathrm{P}$ nanowires display a broad size distribution, consistent with the FESEM observations. From the

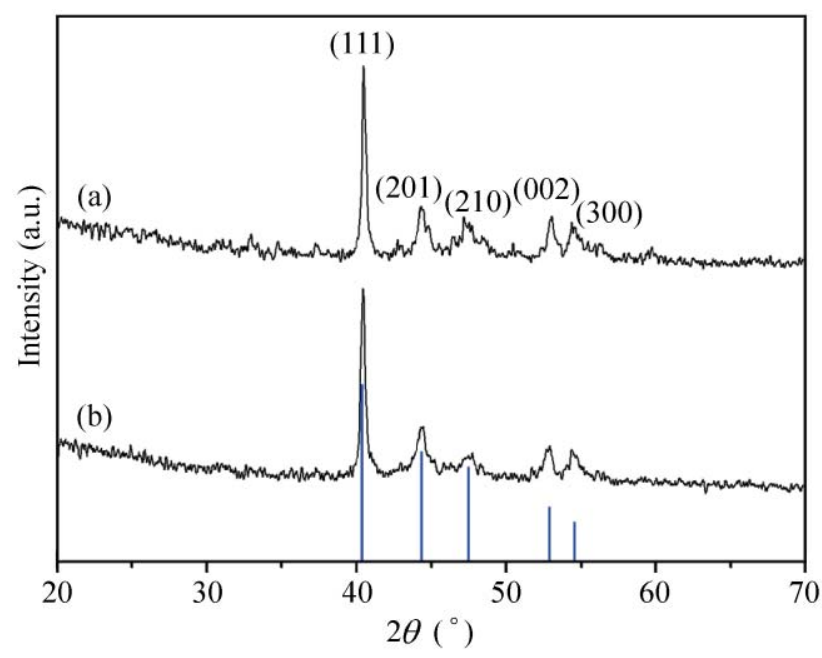

Figure 1 XRD patterns: (a) $\mathrm{Fe}_{2} \mathrm{P}$ nanowires, (b) $\mathrm{Fe}_{2} \mathrm{P} @ \mathrm{C}$ core $@$ shell nanocables 

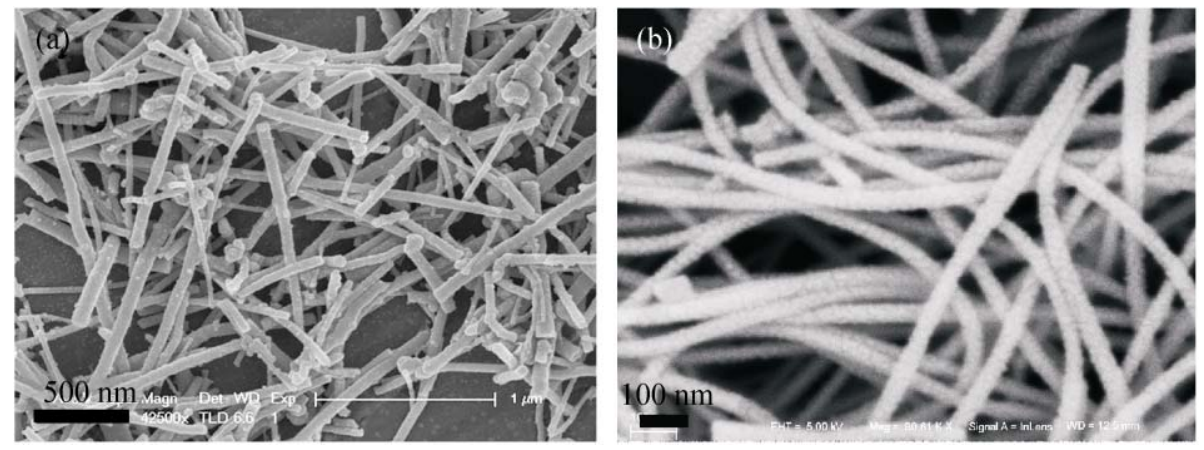

Figure 2 SEM images: (a) $\mathrm{Fe}_{2} \mathrm{P}$ nanowires, (b) $\mathrm{Fe}_{2} \mathrm{P} @ \mathrm{C}$ nanocables
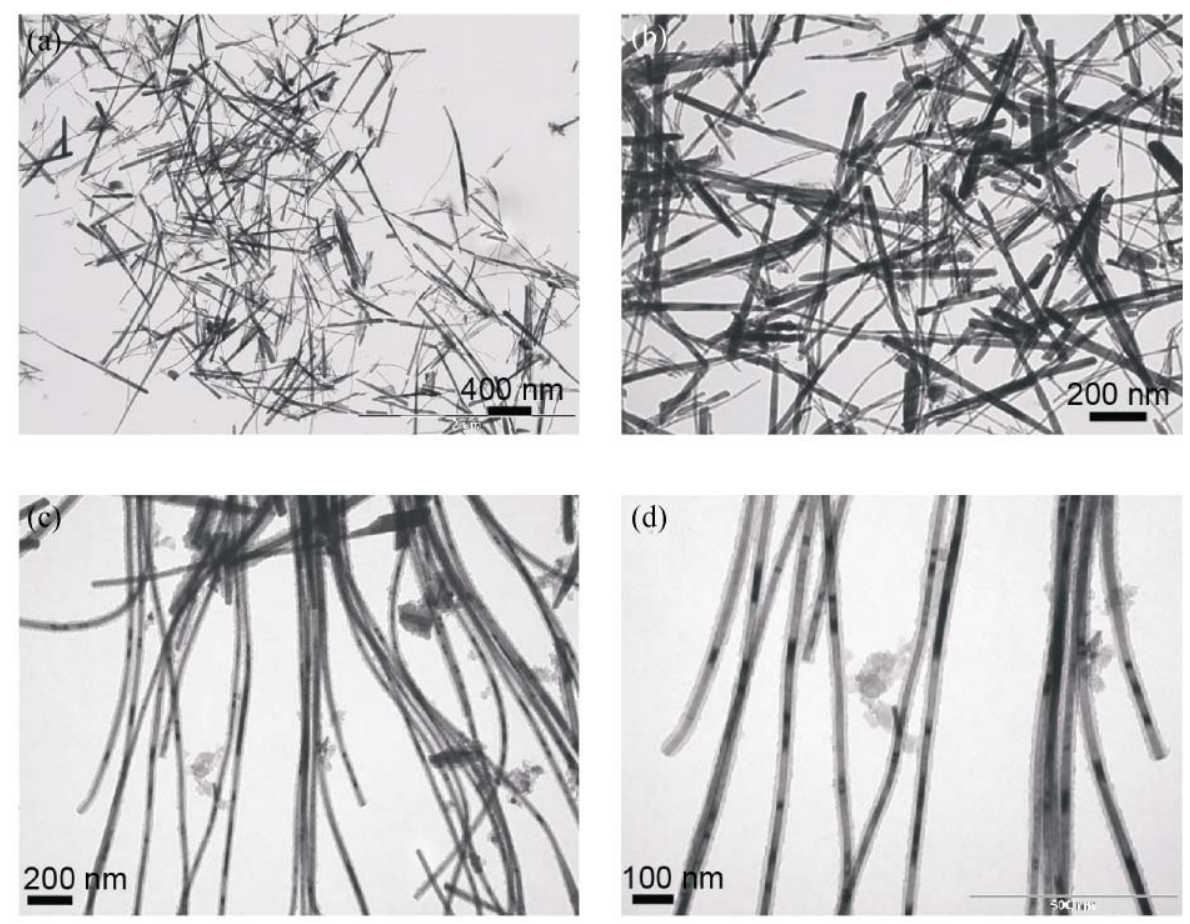

Figure 3 TEM images: (a) and (b) $\mathrm{Fe}_{2} \mathrm{P}$ nanowires, (c) and (d) $\mathrm{Fe}_{2} \mathrm{P} @ \mathrm{C}$ nanocables

TEM studies, one can clearly see that the reaction of Fe powder with $\mathrm{PPh}_{3}$ yields bare $\mathrm{Fe}_{2} \mathrm{P}$ nanowires with no carbon sheath being present on the outer surface of the nanowires. TEM images of $\mathrm{Fe}_{2} \mathrm{P}$ nanocables (Figs. 3(c) and 3(d)) clearly show dark/light contrasts along the radial direction and the different contrasts indicate the coaxial core@shell cable structure. TEM images show that the $\mathrm{Fe}_{2} \mathrm{P}$ nanowire cores of the nanocables have a narrow diameter distribution with a mean diameter of about $20 \mathrm{~nm}$ (Figs. 3(c) and 3(d)).

Figures 4(a)-4(c) show some high-magnification TEM images of the $\mathrm{Fe}_{2} \mathrm{P} @ \mathrm{C}$ nanocables, and further reveal the coaxial core@shell nanostructures of the $\mathrm{Fe}_{2} \mathrm{P} @ \mathrm{C}$ products. EDX spectrometry was used to examine the chemical composition of the nanocables. The EDX spectra taken on a single $\mathrm{Fe}_{2} \mathrm{P} @ \mathrm{C}$ nanocable from the shell to the core testify that the nanocables are composed of only $\mathrm{Fe}, \mathrm{P}$, and $\mathrm{C}$, and the Fe:P ratio in the nanowire core is very close to the stoichiometric value of two (Fig. 4(d)). HRTEM and selected area electron diffraction (SAED) techniques were used to study the microstructures and growth directions of the nanowires and nanocables (Fig. 5). The SAED and HRTEM analyses of $\mathrm{Fe}_{2} \mathrm{P}$ nanowires reveal a lattice spacing of $3.46 \AA$, corresponding to the interplanar distance of (001) planes of hexagonal $\mathrm{Fe}_{2} \mathrm{P}$, indicating 

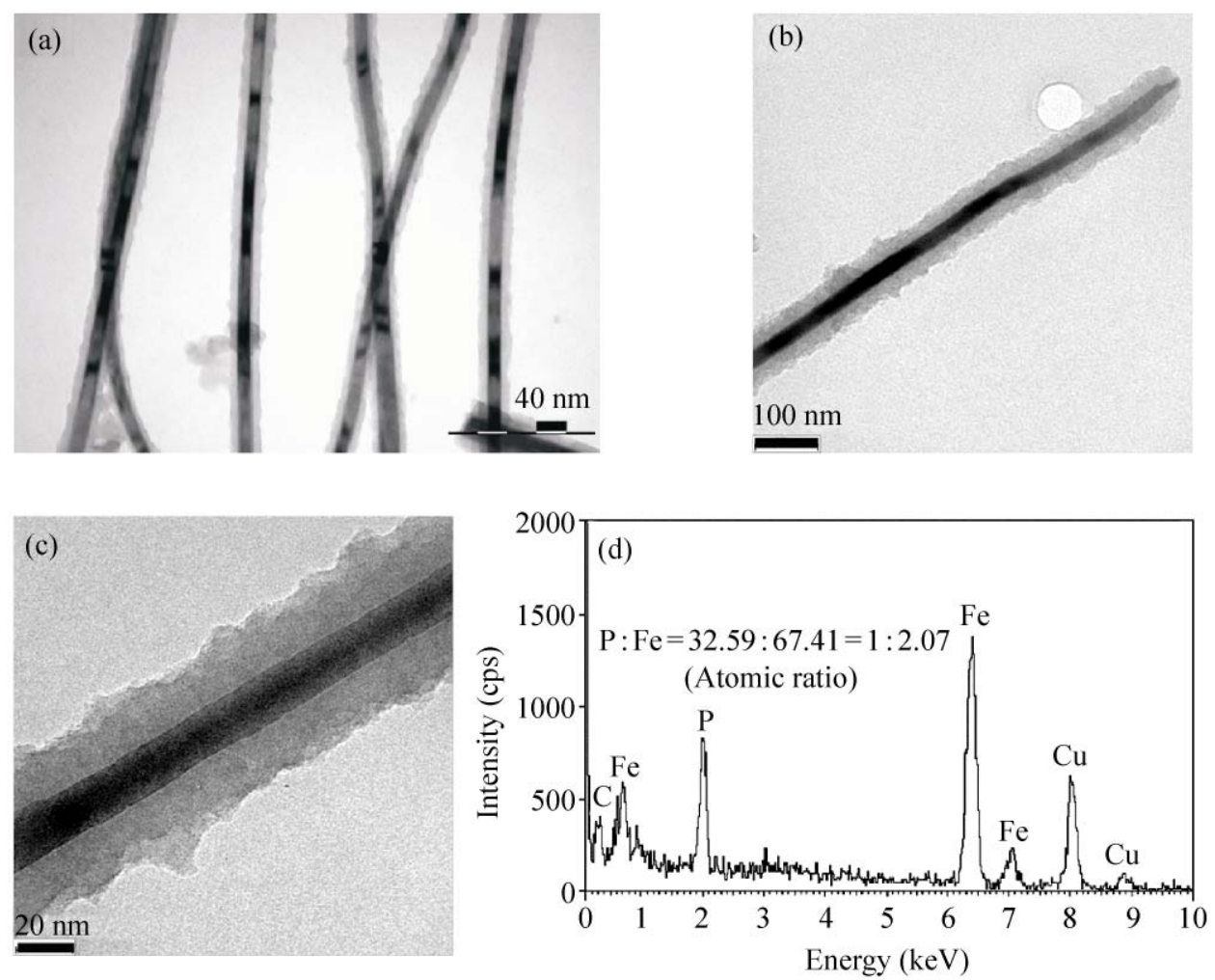

Figure 4 (a), (b), and (c) High-magnification TEM images of $\mathrm{Fe}_{2} \mathrm{P} @ \mathrm{C}$ nanocables; (d) EDX spectrum of the core part of $\mathrm{Fe}_{2} \mathrm{P} @ \mathrm{C}$ nanocables

that $\mathrm{Fe}_{2} \mathrm{P}$ nanowires grow along the [001] direction (Fig. 5(a)). HRTEM studies of $\mathrm{Fe}_{2} \mathrm{P} @ \mathrm{C}$ nanocables (Fig. 5(b)) also demonstrate that the $\mathrm{Fe}_{2} \mathrm{P}$ nanowire core grows along the [001] direction of the hexagonal $\mathrm{Fe}_{2} \mathrm{P}$ structure. The preferred growth direction of the resulting 1-D Fe $\mathrm{P}_{2} \mathrm{P}$ nanostructures is consistent with the previously reported cases [22, 23, 28, 37, 38]. Meanwhile,
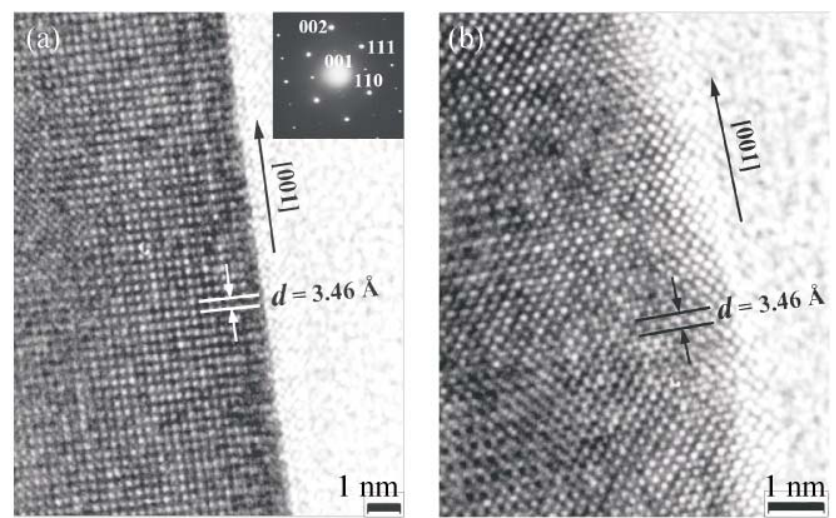

Figure 5 (a) HRTEM image and SAED pattern (in the inset) of $\mathrm{Fe}_{2} \mathrm{P}$ nanowires; (b) HRTEM image of $\mathrm{Fe}_{2} \mathrm{P} @ \mathrm{C}$ nanocables
HRTEM and SAED studies show the high-quality and single crystal nature of the as-obtained nanowires and nanocables.

In our experiments, two kinds of Fe precursors, commercial elemental iron powder and a molecular iron precursor (ferrocene, $\mathrm{Fe}\left(\mathrm{C}_{5} \mathrm{H}_{5}\right)_{2}$ ), were separately used to prepare $\mathrm{Fe}_{2} \mathrm{P}$ nanostructures. Scanning electron microscopy (SEM) and TEM studies (Figs. 2 and 3) show that the size of the products is strongly affected by the iron precursor. The molecular iron precursor $\left(\mathrm{Fe}\left(\mathrm{C}_{5} \mathrm{H}_{5}\right)_{2}\right)$ facilitates diameter control and leads to diameter-uniform $\mathrm{Fe}_{2} \mathrm{P} @ \mathrm{C}$ nanocables, whereas the use of iron powder results in a wide size distribution of the $\mathrm{Fe}_{2} \mathrm{P}$ nanowires. The results are consistent with previously reported work, in which size-uniform 1-D nanocrystals were prepared from molecular metal precursors by the solution-based precursor injection method [14, 15, 22-26, 38]. Some prior studies have revealed that the size and size distribution of metal particles strongly affect those of the final products [24, 34-36]. The Fe powder employed

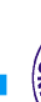

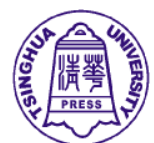

黑 Springer 
was composed of micrometer- or sub-micrometer-scale particles of a wide size distribution, which results in $\mathrm{Fe}_{2} \mathrm{P}$ nanowires with a wide size range when it is converted into $\mathrm{Fe}_{2} \mathrm{P}$.

As shown in Fig. 6, the changes in physical state and color in the sealed ampoule at different reaction stages can be monitored. Figures 6(a), 6(b), and 6(c) show views of the reaction between Fe powder and $\mathrm{PPh}_{3}$. At a temperature of $220^{\circ} \mathrm{C}, \mathrm{Fe}$ is still in the solid state, while $\mathrm{PPh}_{3}$ becomes liquid and colorless. The presence of a red-yellow liquid indicates the occurrence of the reaction between $\mathrm{Fe}$ and $\mathrm{PPh}_{3}$ after heating at $380^{\circ} \mathrm{C}$ for $15 \mathrm{~h}$. When heated at a temperature $\left(380-400{ }^{\circ} \mathrm{C}\right)$ above the boiling point of $\mathrm{PPh}_{3}\left(377^{\circ} \mathrm{C}\right), \mathrm{PPh}_{3}$ molecules will be in the liquid and vapor states and form a reflux medium in the sealed ampoule used for growth of $\mathrm{Fe}_{2} \mathrm{P}$. The chemical reactivity of gas phase $\mathrm{PPh}_{3}$ molecules will be higher than that of solid state or liquid $\mathrm{PPh}_{3}$, which enhances the interaction of $\mathrm{Fe}$ and $\mathrm{P}$ to generate $\mathrm{Fe}_{2} \mathrm{P}$. In the $\mathrm{Fe}_{2} \mathrm{P} @ \mathrm{C}$ nanocable synthesis, $\mathrm{Fe}\left(\mathrm{C}_{5} \mathrm{H}_{5}\right)_{2}$ and $\mathrm{PPh}_{3}$ are in the solid state at room temperature before heating (Fig. 6(a')). When heated at a temperature of $220{ }^{\circ} \mathrm{C}$, which is higher than the melting points of $\mathrm{Fe}\left(\mathrm{C}_{5} \mathrm{H}_{5}\right)_{2}$ and $\mathrm{PPh}_{3}$ (Table 1), the reactants become molten and a red transparent homogeneous liquid is formed (Fig. 6(b')), since $\mathrm{Fe}\left(\mathrm{C}_{5} \mathrm{H}_{5}\right)_{2}$ and $\mathrm{PPh}_{3}$ are completely mutually soluble in the liquid state owing to their aromatic characteristics. In general, Refs. [14, 15, 21-28, 38] suggest that the formation of a homogeneous solution of reactants favors the growth of size-uniform nanocrystals. When the vacuum- sealed ampoule is quickly transferred into a $400{ }^{\circ} \mathrm{C}$ furnace from the $220{ }^{\circ} \mathrm{C}$ furnace, the reaction between $\mathrm{Fe}\left(\mathrm{C}_{5} \mathrm{H}_{5}\right)_{2}$ and $\mathrm{PPh}_{3}$ progresses very rapidly, and a black product of $\mathrm{Fe}_{2} \mathrm{P} @ \mathrm{C}$ nanocables results (Fig. 6(c')).

These experimental results show that the temperature sudden rise leads to a rapid thermal decomposition of $\mathrm{Fe}\left(\mathrm{C}_{5} \mathrm{H}_{5}\right)_{2}$ in the vacuum-sealed ampoules. The rapid decomposition of $\mathrm{Fe}\left(\mathrm{C}_{5} \mathrm{H}_{5}\right)_{2}$ yields a large quantity of uniform Fe nanoparticles with high activity in the early stages of the reaction due to the fast homogeneous nucleation process and the newly formed uniform Fe nanoparticles are easily converted into $\mathrm{Fe}_{2} \mathrm{P}$ through solution-mediated reaction with liquid $\mathrm{PPh}_{3}$ at elevated temperatures; this provides a good platform for the growth of diameter-uniform $\mathrm{Fe}_{2} \mathrm{P} @ \mathrm{C}$ nanocables. As shown in Fig. 7(a), the XRD and TEM studies provide evidence for the thermal decomposition of $\mathrm{Fe}\left(\mathrm{C}_{5} \mathrm{H}_{5}\right)_{2}$. The two strong diffraction peaks in the XRD patterns can be assigned to $\mathrm{Fe}_{2} \mathrm{P}(111)$ and $\mathrm{Fe}(110)$ planes. From the XRD pattern, it is noted that the relative intensity of the $\mathrm{Fe}(110)$ peak is larger than that of the $\mathrm{Fe}_{2} \mathrm{P}(111)$ peak, which shows that the content of pure $\mathrm{Fe}$ is quite high at this early reaction stage. To further investigate the reaction mechanism, $2 \mathrm{mmol}$ of $\mathrm{Fe}\left(\mathrm{C}_{5} \mathrm{H}_{5}\right)_{2}$ was heated at $400{ }^{\circ} \mathrm{C}$ for $40 \mathrm{~min}$ in the evacuated and sealed ampoule during the temperature sudden rise process and the resulting product was analyzed by TEM and EDX (shown in Figs. 7(b) and 7(c)). The TEM image shows the production of Fe nanoparticles with an average diameter of $8 \mathrm{~nm}$, and the EDX spectrum taken of the Fe nanoparticles confirms the presence of iron and absence of phosphorus in the product.

Besides the influence of the Fe precursor, the diameter control is also affected by the specific nucleation and growth mechanism of the 1-D $\mathrm{Fe}_{2} \mathrm{P}$ nanostructures. The formation of nanocrystals usually takes place via two stages: nucleation and growth $[11,14,15,39]$. It is considered that the suddentemperature-rise process in our synthesis plays similar and important roles in the nucleation of target

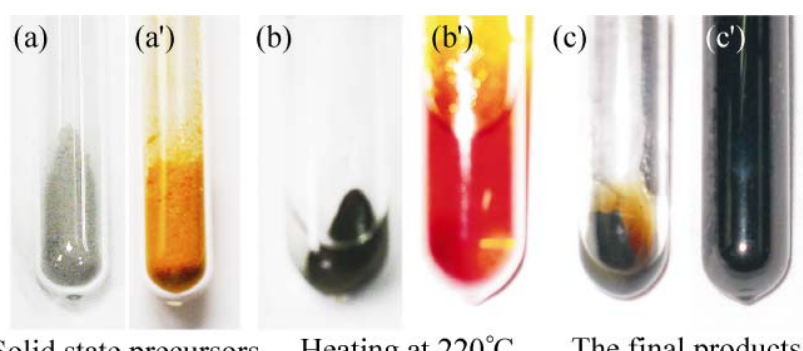

Solid state precursors Heating at $220^{\circ} \mathrm{C}$ The final products

Figure 6 The changes of state and color at the different stages of the synthesis: (a) and (a') the solid state precursors at room temperature; (b) and (b') the precursors heated at $220{ }^{\circ} \mathrm{C}$; (c) and (c') the final products after reaction; (a), (b), and (c) indicate the changes during the synthesis of $\mathrm{Fe}_{2} \mathrm{P}$ nanowires; (a'), (b'), and (c') indicate those for $\mathrm{Fe}_{2} \mathrm{P} @ \mathrm{C}$ nanocables

Table 1 Melting points of the precursors employed in the syntheses

\begin{tabular}{cccc}
\hline Substance & $\mathrm{Fe}$ & $\mathrm{Fe}\left(\mathrm{C}_{5} \mathrm{H}_{5}\right)_{2}$ & $\mathrm{PPh}_{3}$ \\
\hline Melting point $\left({ }^{\circ} \mathrm{C}\right)$ & 1535 & 174 & 80 \\
\hline
\end{tabular}



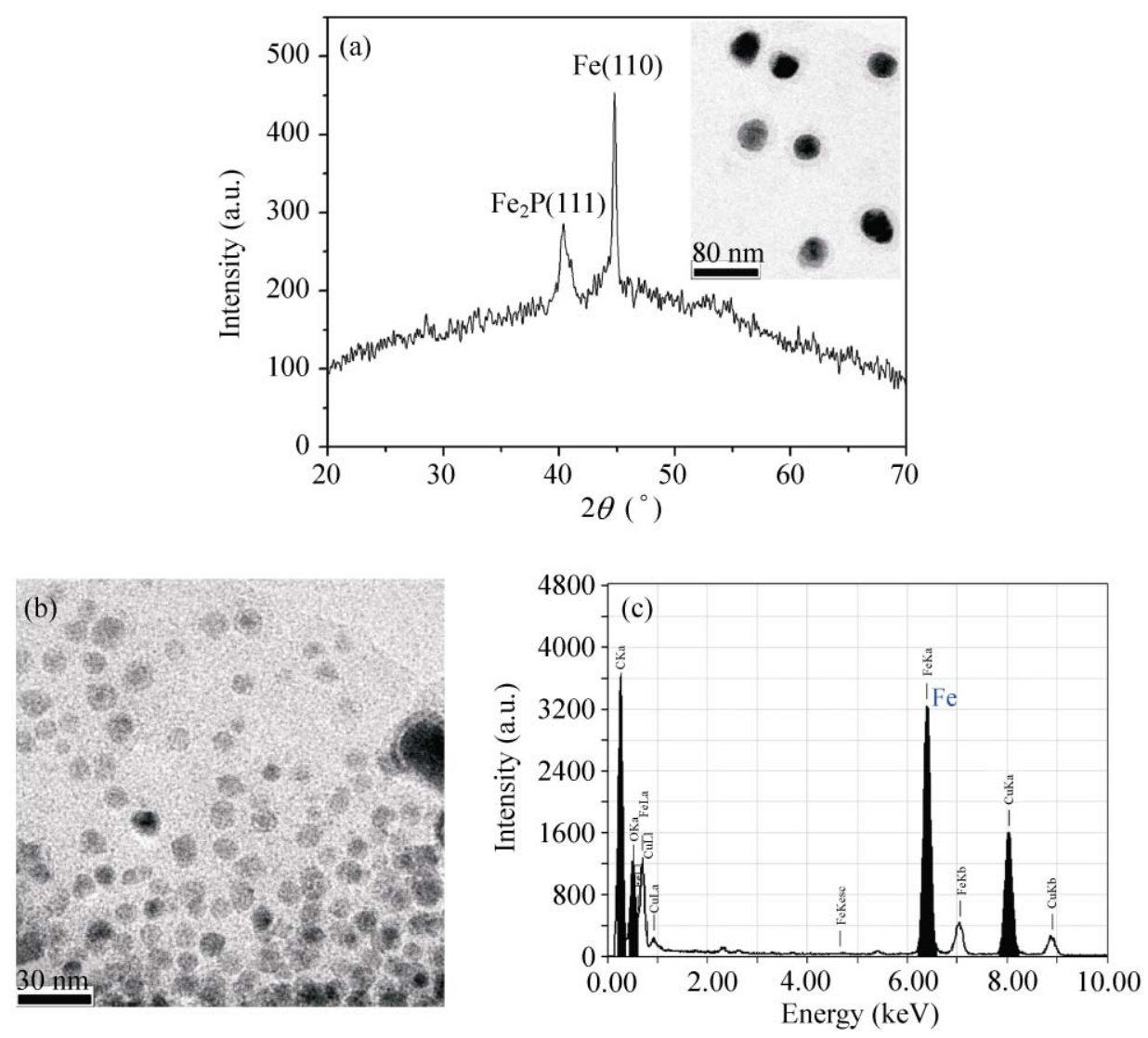

Figure 7 (a) XRD pattern and TEM image (in the inset) of the products obtaine $\mathrm{d}$ from the reaction of ferrocene with $\mathrm{PPh}_{3}$ at a temperature of $400{ }^{\circ} \mathrm{C}$ for $40 \mathrm{~min}$, revealing the presence of metallic $\mathrm{Fe}$ and $\mathrm{Fe}_{2} \mathrm{P}$ at the early reaction stage of the $\mathrm{Fe}_{2} \mathrm{P} @ \mathrm{C}$ nanocable synthesis; (b) TEM image of Fe nanoparticles produced by thermal decomposition of $\mathrm{Fe}\left(\mathrm{C}_{5} \mathrm{H}_{5}\right)_{2}$ at $400{ }^{\circ} \mathrm{C}$ after 40 min in the sudden-temperature-rise process; (c) EDX spectrum of the Fe nanoparticles in (b)

nanocrystals to those of the rapid-injection-of-precursor process in solution-based precursor injection methods $[14,15,22-26]$, based on the fact that both processes can result in a burst and uniform nucleation of nanocrystals. In the growth of $\mathrm{Fe}_{2} \mathrm{P} @ \mathrm{C}$ nanocables from molecular precursors, our investigations suggest that the nucleation generally progresses separately from the growth stage. When the reaction temperature rises suddenly from $220^{\circ} \mathrm{C}$ to $400{ }^{\circ} \mathrm{C}$, a large number of small uniform Fe nanoparticles with high reactivity are produced in a short period of time by rapid decomposition of $\mathrm{Fe}\left(\mathrm{C}_{5} \mathrm{H}_{5}\right)_{2}$ in the vacuum-sealed ampoule. The presence of this large number of small $\mathrm{Fe}$ nanoparticles favors the growth of an Fe-rich $\mathrm{Fe}_{2} \mathrm{P}$ phase, and the size-uniform Fe nanoparticles with their high surface energy will quickly interact with reactive liquid $\mathrm{PPh}_{3}$ to form a large number of uniform $\mathrm{Fe}_{2} \mathrm{P}$ seeds at a fast rate. In this way, a burst nucleation $[14,15,39,40]$ of $\mathrm{Fe}_{2} \mathrm{P}$ with uniform sizes can be realized in a short period of time by the sudden rise of reaction temperature. The size-uniform $\mathrm{Fe}_{2} \mathrm{P}$ seeds favor the subsequent growth of $\mathrm{Fe}_{2} \mathrm{P} @ \mathrm{C}$ nanocables with uniform sizes.

As for the synthesis of $\mathrm{Fe}_{2} \mathrm{P}$ nanowires, the nucleation of $\mathrm{Fe}_{2} \mathrm{P}$ on high-melting metallic Fe particles is one type of heterogeneous nucleation and will produce many $\mathrm{Fe}_{2} \mathrm{P}$ nuclei or seeds with different sizes derived from the wide size distribution of the Fe powder, because the variously sized particles have different surface energies and nucleation abilities [24, 34-36]. Moreover, secondary nucleation of $\mathrm{Fe}_{2} \mathrm{P}$ also occurs on the surfaces of $\mathrm{Fe}$ particles to form new $\mathrm{Fe}_{2} \mathrm{P}$ nanowires during the growth of the initially formed nanowires. That is to say, the nucleation and growth of $\mathrm{Fe}_{2} \mathrm{P}$ nanocrystals occur over a long period of time in the formation of $\mathrm{Fe}_{2} \mathrm{P}$ nanowires, and the separation 
between nucleation and growth cannot be achieved by a sudden rise in temperature. As a consequence, $\mathrm{Fe}_{2} \mathrm{P}$ nanowires with a broad size distribution result.

The growth mechanism of $\mathrm{Fe}_{2} \mathrm{P}$ nanowires and $\mathrm{Fe}_{2} \mathrm{P} @ \mathrm{C}$ nanocables can be reasonably attributed to the cooperative effects of the kinetically induced anisotropic growth and the intrinsically anisotropic crystal structure of hexagonal $\mathrm{Fe}_{2} \mathrm{P}$. The $\mathrm{Fe}-\mathrm{P}$ phase diagram [41] and high melting point of Fe show that no eutectic alloy or liquid metal catalyst can be produced in the reaction at $380-400{ }^{\circ} \mathrm{C}$, so the metalcatalyzed vapor-liquid-solid (VLS) or solution-liquidsolid (SLS) growth mechanisms [1, 2, 14, 17-20] are excluded for the formation of $1-\mathrm{D} \mathrm{Fe}_{2} \mathrm{P}$ nanostructures synthesized by our method. It is noted that our reaction system is very simple, with $\mathrm{PPh}_{3}$ being utilized as both stabilizing ligand and phosphorus source and a mixture of surfactants or a precursor injection is not required for anisotropic crystal growth. Thus, the selective-adsorption model of surfactants [8-11, 14, $15,24]$, which is often used to explain the formation of 1-D nanocrystals, is not applicable to the formation of $\mathrm{Fe}_{2} \mathrm{P}$ nanowires or $\mathrm{Fe}_{2} \mathrm{P} @ \mathrm{C}$ nanocables. In the current syntheses, we note that the crystalline-seed phase of $\mathrm{Fe}_{2} \mathrm{P}$ has a hexagonal structure, and therefore $\mathrm{Fe}_{2} \mathrm{P}$ nanocrystals intrinsically tend to be elongated in the [001] direction due to the high surface energy of the crystallographic $\{001\}$ faces of the hexagonal structure $[11,14,38]$. Meanwhile, the solution-mediated reaction between Fe particles and $\mathrm{PPh}_{3}$ at temperatures of 380-400 ${ }^{\circ} \mathrm{C}$ offers a suitable kinetic growth regime. In the growth of 1-D $\mathrm{Fe}_{2} \mathrm{P}$ nanocrystals, the diffusion between $\mathrm{Fe}$ and $\mathrm{P}$ atoms occurs at a considerable rate in liquid $\mathrm{PPh}_{3}$, and a high flux of conversion of $\mathrm{Fe}_{2} \mathrm{P}$ monomers into initial $\mathrm{Fe}_{2} \mathrm{P}$ seeds is created via diffusional growth [11, 14, 39, 40], which favors the preferential 1-D growth of monomers on the highest energy (001) surface [11, 14, 39], and finally yields the 1-D $\mathrm{Fe}_{2} \mathrm{P}$ nanostructures grown along the [001] direction (as verified by HRTEM and SAED studies). As the kinetic-dominating growth regime will be destroyed by adding extra surfactants or stabilizing ligands, the shape of the $\mathrm{Fe}_{2} \mathrm{P}$ products will be changed. In such an experiment, we added triphenylphosphine oxide $\left(\mathrm{OPPh}_{3}\right)$ to the mixed reactants of $\mathrm{Fe}\left(\mathrm{C}_{5} \mathrm{H}_{5}\right)_{2}$ and $\mathrm{PPh}_{3}$, whereupon $\mathrm{Fe}_{2} \mathrm{P}$ and $\mathrm{C}$ nanoparticles, rather than nanocables, were produced (Fig. S-1 in the in the Electronic Supplementary Material (ESM)).

The magnetic properties of the as-synthesized 1-D $\mathrm{Fe}_{2} \mathrm{P}$ nanostructures were measured on an SQUID magnetometer. Figure 8 shows the temperature dependence of ZFC and FC magnetization for $\mathrm{Fe}_{2} \mathrm{P}$ nanowires and $\mathrm{Fe}_{2} \mathrm{P} @ \mathrm{C}$ nanocables. As shown in Fig. 8(a), in the ZFC mode for the $\mathrm{Fe}_{2} \mathrm{P}$ nanowires, the magnetization increases at first, and then decreases with increasing temperature. The blocking temperature $\left(T_{\mathrm{B}}\right)$ for the as-synthesized $\mathrm{Fe}_{2} \mathrm{P}$ nanowires is $230 \mathrm{~K}$ under an applied magnetic field of $100 \mathrm{Oe}$, which is close to the literature data $[23,31]$. The $\mathrm{Fe}_{2} \mathrm{P}$ nanowires display a ferromagnetic-paramagnetic transition at $230 \mathrm{~K}[23,31,33]$. Below the blocking temperature, the $\mathrm{Fe}_{2} \mathrm{P}$ nanowires are ferromagnetic and display hysteresis behavior. In the hysteresis loops (the inset of Fig. 8(a)), the coercive field is as large as 7000 Oe at $5 \mathrm{~K}$, whilst no discernible hysteretic behavior was observed at $300 \mathrm{~K}$. In the hysteresis loops, the magnetization of $\mathrm{Fe}_{2} \mathrm{P}$ nanowires was not saturated with an external field of $2 \mathrm{~T}$, which also implies the as-synthesized $\mathrm{Fe}_{2} \mathrm{P}$ nanowire sample is a combination of paramagnetic and ferromagnetic constituents [31].

The magnetic behaviors (Fig. 8(b)) of the $\mathrm{Fe}_{2} \mathrm{P} @ \mathrm{C}$ nanocables synthesized in a typical process are similar to those of the $\mathrm{Fe}_{2} \mathrm{P}$ nanowires, because the magnetic properties of the both samples are derived from $\mathrm{Fe}_{2} \mathrm{P}$. In the ZFC mode (Fig. 8(b)) the blocking temperature is $268 \mathrm{~K}$, close to that of a sample synthesized with higher Fe:P ratios [38], and below this temperature the $\mathrm{Fe}_{2} \mathrm{P} @ \mathrm{C}$ nanocable sample displays hysteresis behavior. As shown in the inset of Fig. 8(b), the hysteresis loop at $5 \mathrm{~K}$ has a coercive field of $7400 \mathrm{Oe}$, whilst no discernible hysteretic behavior was observed at $300 \mathrm{~K}$ at an applied magnetic field of 100 Oe. Furthermore, the loops show that the magnetization of $\mathrm{Fe}_{2} \mathrm{P} @ \mathrm{C}$ nanocables is still not saturated even under an external field of $4 \mathrm{~T}$. Figure 8(c) shows the ZFC modes for both the $\mathrm{Fe}_{2} \mathrm{P}$ nanowires and the $\mathrm{Fe}_{2} \mathrm{P} @ \mathrm{C}$ nanocables, from which we can easily see that the $\mathrm{Fe}_{2} \mathrm{P} @ \mathrm{C}$ nanocables have a higher $T_{B}$ than the $\mathrm{Fe}_{2} \mathrm{P}$ nanowires. We note that the blocking temperatures for both $\mathrm{Fe}_{2} \mathrm{P}$ nanowires $\left(T_{\mathrm{B}}=230 \mathrm{~K}\right)$ and $\mathrm{Fe}_{2} \mathrm{P} @ \mathrm{C}$ nanocables $\left(T_{\mathrm{B}}=268 \mathrm{~K}\right)$ are higher than the bulk ferromagnetic transition temperature of $\mathrm{Fe}_{2} \mathrm{P}\left(T_{\mathrm{C}}=217 \mathrm{~K}\right)$ [42]. It is well known that 


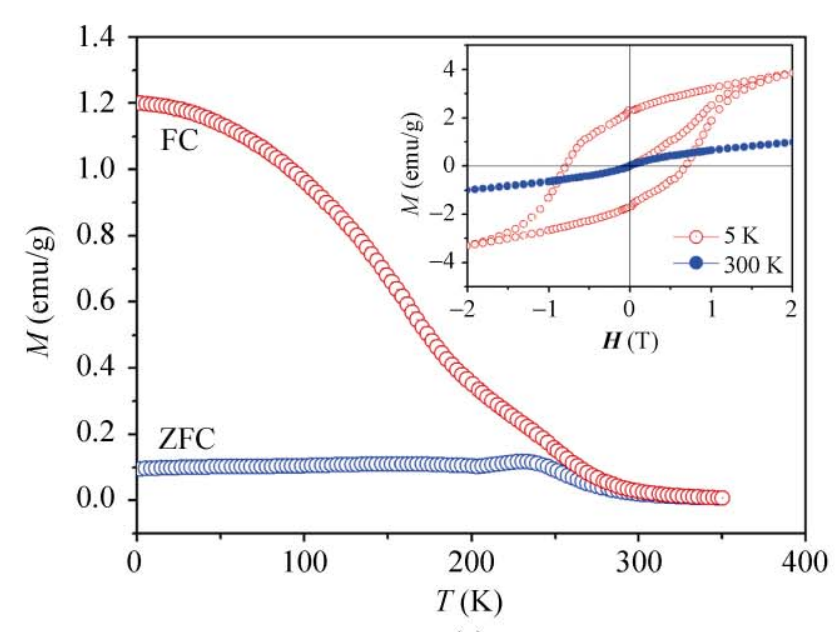

(a)

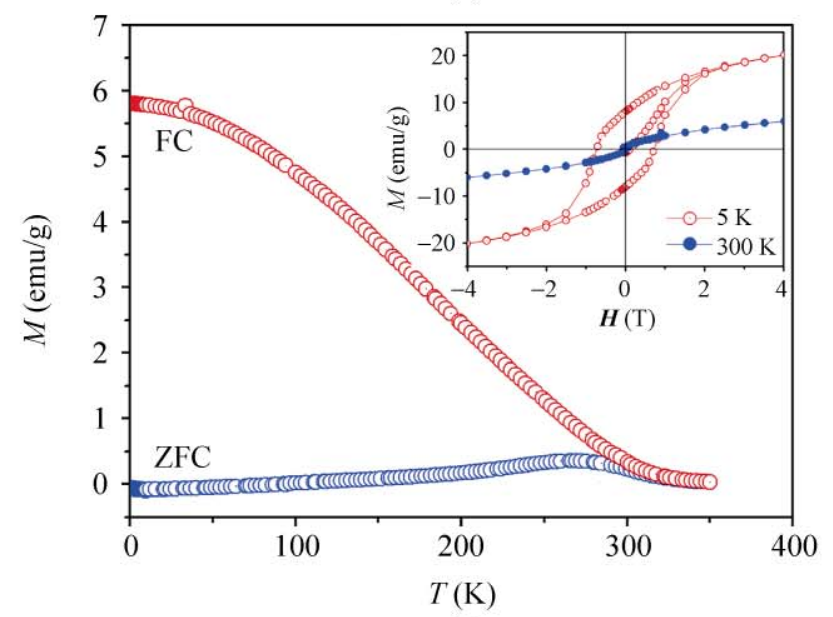

(b)

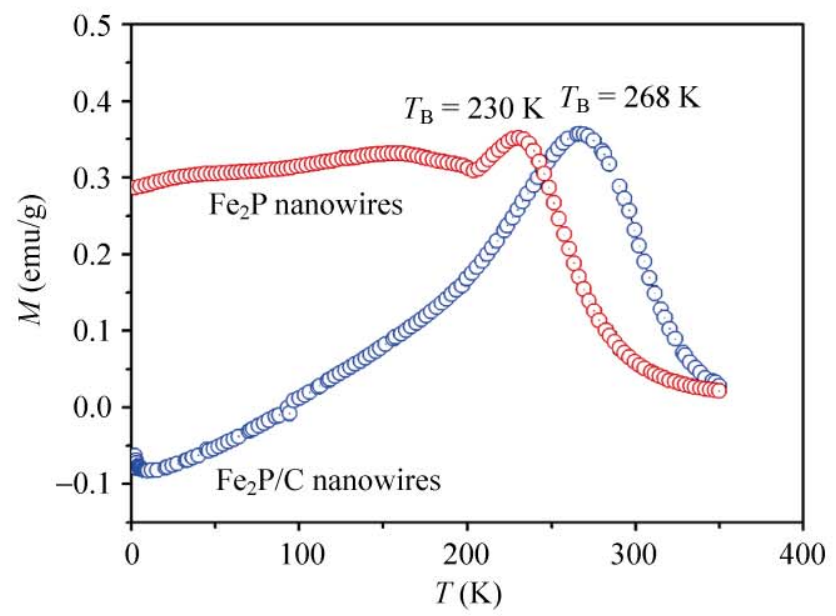

(c)

Figure 8 Plots of ZFC and FC magnetization vs. temperature for samples of (a) $\mathrm{Fe}_{2} \mathrm{P}$ nanowires and (b) $\mathrm{Fe}_{2} \mathrm{P} @ \mathrm{C}$ nanocables prepared in typical syntheses, at an applied magnetic field of $100 \mathrm{Oe}$. The insets display the magnetization hysteresis loops recorded at 5 and $300 \mathrm{~K}$. (c) Plots of ZFC magnetization vs. temperature, clearly showing the variation in blocking temperatures of the 1-D $\mathrm{Fe}_{2} \mathrm{P}$ nanostructures the ferromagnetic transition of $\mathrm{Fe}_{2} \mathrm{P}$ is very sensitive to variations in physical and chemical parameters such as particle size, shape, and composition, since it has strong ferromagnetic spin fluctuations [23, 42]. The higher blocking temperatures of our samples are probably caused by the high aspect ratio (length/width) and the richer Fe concentration (such as the presence of pure $\mathrm{Fe}$ ) than stoichiometric $\mathrm{Fe}_{2} \mathrm{P}$ for the as-synthesized 1-D Fe 2 P nanostructures [22-24, 37, 38].

\section{Conclusions}

$\mathrm{Fe}_{2} \mathrm{P}$ nanowires and $\mathrm{Fe}_{2} \mathrm{P} @ \mathrm{C}$ core@shell nanocables have been synthesized by reactions of $\mathrm{PPh}_{3}$ with elemental Fe powder and a molecular iron precursor of $\mathrm{Fe}\left(\mathrm{C}_{5} \mathrm{H}_{5}\right)_{2}$, respectively. The syntheses were based on the conversion of $\mathrm{Fe}$ particles into $\mathrm{Fe}_{2} \mathrm{P}$ via extracting phosphorus from $\mathrm{PPh}_{3}$ at elevated temperatures. In the synthesis, a novel sudden-temperature-rise strategy was employed to control the product sizes, and we investigated in detail the influences of the iron precursor, temperature-rise rate, and the nucleation and growth mechanism on the diameter control and the formation of the 1-D $\mathrm{Fe}_{2} \mathrm{P}$ nanostructures. The resulting 1-D $\mathrm{Fe}_{2} \mathrm{P}$ nanostructures exhibited ferromagnetic-paramagnetic transition behaviors with high blocking temperatures, and are potential components in nanowire-based magnetic devices. We expect that our work offers an alternative promising method to prepare 1-D phosphide nanostructures, and that the sudden-temperature-rise strategy in vacuum-sealed ampoules can be extended to the shape control of other 1-D nanomaterials.

\section{Acknowledgements}

We gratefully acknowledge the financial support from the K. C. Wong Education Foundation of Hong Kong, the National Natural Science Foundation of China (No. 20571068), the Program for New Century Excellent Talents at Universities from the Chinese Ministry of Education (No. NCET2006-0552), the Foundation of Anhui Provincial Education Department (No. KJ2008A071), the Creative Research Foundation for Graduates of USTC (No. KD2008019), and the 
Chinese Academy of Sciences (CAS) Special Grant for Postgraduate Research, Innovation and Practice (2008).

Electronic Supplementary Material: The XRD pattern and TEM image of the products obtained in the presence of triphenylphosphine oxide $\left(\mathrm{OPPh}_{3}\right)$. This material is available in the online version of this article at http://dx.doi.org/10.1007/s12274-010-1024-2 and is accessible free of charge.

Open Access: This article is distributed under the terms of the Creative Commons Attribution Noncommercial License which permits any noncommercial use, distribution, and reproduction in any medium, provided the original author(s) and source are credited.

\section{References}

[1] Xia, Y. N.; Yang, P. D.; Sun, Y. G.; Wu, Y. Y.; Mayers, B.; Gates, B.; Yin, Y. D.; Kim, F.; Yan, H. Q. One-dimensional nanostructures: Synthesis, characterization, and applications. Adv. Mater. 2003, 15, 353-389.

[2] Law, M.; Goldberger, J.; Yang, P. D. Semiconductor nanowires and nanotubes. Ann. Rev. Mater. Res. 2004, 34, 83-122.

[3] Li, Y.; Qian, F.; Xiang, J.; Lieber, C. M. Nanowire electronic and optoelectronic devices. Mater. Today 2006, 9, 18-27.

[4] Wang, Z. L.; Song, J. H. Piezoelectric nanogenerators based on zinc oxide nanowire arrays. Science 2006, 312, 242-246.

[5] Xu, C.; Wang, X. D.; Wang, Z. L. Nanowire structured hybrid cell for concurrently scavenging solar and mechanical energies. J. Am. Chem. Soc. 2009, 131, 5866-5872.

[6] Aronsson, B.; Lundstrom, T.; Rundquist, S. Borides, Silicides, and Phosphides: A Critical Review of Their Preparation, Properties, and Crystal Chemistry; Ballantyne and Co.: Spottiswoode, U.K., 1965.

[7] Oyama, S. T. Novel catalysts for advanced hydroprocessing: Transition metal phosphides. J. Catal. 2003, 216, 343-352.

[8] Gschneidner Jr. K. A.; Pecharsky, V. K.; Tsokol, A. O. Recent developments in magnetocaloric materials. Rep. Prog. Phys. 2005, 68, 1479-1539.

[9] Gillot, F.; Boyanov, S.; Dupont, L.; Doublet, M. L.; Morcrette, M.; Monconduit, L.; Tarascon, J. M. Electrochemical reactivity and design of $\mathrm{NiP}_{2}$ negative electrodes for secondary Li-ion batteries. Chem. Mater. 2005, 17, 6327-6337.

[10] Boyanov, S.; Bernardi, J.; Gillot, F.; Dupont, L.; Womes, M.; Tarascon, J. M.; Monconduit, L.; Doublet, M. L. FeP: Another attractive anode for the Li-ion battery enlisting a reversible two-step insertion/conversion process. Chem. Mater. 2006, 18, 3531-3538.

[11] Xia, Y. N.; Xiong, Y. J.; Lim, B.; Skrabalak, S. E. Shapecontrolled synthesis of metal nanocrystals: Simple chemistry meets complex physics? Angew. Chem. Int. Ed. 2009, 48, 60-103.

[12] Sun, S. H.; Murray, C. B.; Weller, D.; Folks, L.; Moser, A. Monodisperse FePt nanoparticles and ferromagnetic FePt nanocrystal superlattices. Science 2000, 287, 1989-1992.

[13] Comini, E.; Baratto, C.; Faglia, G.; Ferroni, M.; Vomiero, A.; Sberveglieri, G. Quasi-one-dimensional metal oxide semiconductors: Preparation, characterization and application as chemical sensors. Prog. Mater. Sci. 2009, 54, 1-67.

[14] Jun, Y.; Choi, J.; Cheon, J. Shape control of semiconductor and metal oxide nanocrystals through nonhydrolytic colloidal routes. Angew. Chem. Int. Ed. 2006, 45, 3414-3439.

[15] Peng, X. G.; Wickham, J.; Alivisatos, A. P. Kinetics of IIVI and III $-\mathrm{V}$ colloidal semiconductor nanocrystal growth: "Focusing" of size distributions. J. Am. Chem. Soc. 1998, 120, 5343-5344.

[16] Wang, X.; Zhuang, J.; Peng, Q.; Li, Y. D. A general strategy for nanocrystal synthesis. Nature 2005, 437, 121-124.

[17] Homma, Y.; Liu, H. P.; Takagi, D.; Kobayashi, Y. Singlewalled carbon nanotube growth with non-iron-group "catalysts" by chemical vapor deposition. Nano Res. 2009, 2, 793-799.

[18] Kodambaka, S.; Tersoff, J.; Reuter, M. C.; Ross, F. M. Germanium nanowire growth below the eutectic temperature. Science 2007, 316, 729-732.

[19] Wang, F.; Buhro, W. E. Determination of the rod-wire transition length in colloidal indium phosphide quantum rods. J. Am. Chem. Soc. 2007, 129, 14381-14387.

[20] Fanfair, D. D.; Korgel, B. A. Bismuth nanocrystal-seeded III $-\mathrm{V}$ semiconductor nanowire synthesis. Cryst. Growth Des. 2005, 5, 1971-1976.

[21] Brock, S. L.; Perera, S. C.; Stamm, K. L. Chemical routes for production of transition metal phosphides on the nanoscale: Implications for advanced magnetic and catalytic materials. Chem. Eur. J. 2004, 10, 3364-3371.

[22] Park, J.; Koo, B.; Yoon, K. Y.; Hwang, Y.; Kang, M.; Park, J. G.; Hyeon, T. Generalized synthesis of metal phosphide nanorods via thermal decomposition of continuously delivered metal-phosphine complexes using a syringe pump. $\mathrm{J}$. Am. Chem. Soc. 2005, 127, 8433-8440.

[23] Park, J.; Koo, B.; Hwang, Y.; Bae, C.; An, K.; Park, J. G.; Park, H. M.; Hyeon, T. Novel synthesis of magnetic $\mathrm{Fe}_{2} \mathrm{P}$ nanorods from thermal decomposition of continuously delivered precursors using a syringe pump. Angew. Chem., Int. Ed. 2004, 43, 2282-2285. 
[24] Chen, J. H.; Taib, M. F.; Chi, K. M. Catalytic synthesis, characterization and magnetic properties of iron phosphide nanowires. J. Mater. Chem. 2004, 14, 296-298.

[25] Qian, C.; Kim, F.; Ma, L.; Tsui, F.; Yang, P. D.; Liu, J. Solution-phase synthesis of single-crystalline iron phosphide nanorods/nanowires. J. Am. Chem. Soc. 2004, 126, 1195-1198.

[26] Gregg, K. A.; Perera, S. C.; Lawes, G.; Shinozaki, S.; Brock, S. L. Controlled synthesis of MnP nanorods: Effect of shape anisotropy on magnetization. Chem. Mater. 2006, 18, 879-886.

[27] Li, Y.; Malik, M. A.; O'Brien, P. Synthesis of singlecrystalline $\mathrm{CoP}$ nanowires by a one-pot metal-organic route. J. Am. Chem. Soc. 2005, 127, 16020-16021.

[28] Kelly, A. T.; Rusakova, I.; Ould-Ely, T.; Hofmann, C.; Lüttge, A.; Whitmire, K. H. Iron phosphide nanostructures produced from a single-source organometallic precursor: Nanorods, bundles, crosses, and spherulites. Nano Lett. 2007, 7, 2920-2925.

[29] Lukehart, C. M.; Milne, S. B.; Stock, S. R. Formation of crystalline nanoclusters of $\mathrm{Fe}_{2} \mathrm{P}, \mathrm{RuP}, \mathrm{Co}_{2} \mathrm{P}, \mathrm{Rh}_{2} \mathrm{P}, \mathrm{Ni}_{2} \mathrm{P}$, $\mathrm{Pd}_{5} \mathrm{P}_{2}$, or $\mathrm{PtP}_{2}$ in a silica xerogel matrix from single-source molecular precursors. Chem. Mater. 1998, 10, 903-908.

[30] Xie, Y.; Su, H. L.; Qian, X. F.; Liu, X. M.; Qian, Y. T. A mild one-step solvothermal route to metal phosphides (metal $=$ Co, Ni, Cu). J. Solid State Chem. 2000, 149, 88-91.

[31] Luo, F.; Su, H. L.; Song, W.; Wang, Z. M.; Yan, Z. G.; Yan, C. H. Magnetic and magnetotransport properties of $\mathrm{Fe}_{2} \mathrm{P}$ nanocrystallites via a solvothermal route. J. Mater. Chem. 2004, 14, 111-115.

[32] Perera, S. C.; Tsoi, G.; Wenger, L. E.; Brock, S. L. Synthesis of MnP nanocrystals by treatment of metal carbonyl complexes with phosphines: A new, versatile route to nanoscale transition metal phosphides. J. Am. Chem. Soc. 2003, 125, 13960-13961.

[33] Stamm, K. L.; Garno, J. C.; Liu, G. Y.; Brock, S. L. A general methodology for the synthesis of transition metal pnictide nanoparticles from pnictate precursors and its application to iron-phosphorus phases. J. Am. Chem. Soc. 2003, 125, 4038-4039.

[34] Chiang, R. K.; Chiang, R. T. Formation of hollow $\mathrm{Ni}_{2} \mathrm{P}$ nanoparticles based on the nanoscale Kirkendall effect. Inorg. Chem. 2007, 46, 369-371.

[35] Henkes, A. E.; Vasquez, Y.; Schaak, R. E. Converting metals into phosphides: A general strategy for the synthesis of metal phosphide nanocrystals. J. Am. Chem. Soc. 2007, 129, 1896-1897.

[36] Vasquez, Y.; Henkes, A. E.; Bauer, J. C.; Schaak, R. E. Nanocrystal conversion chemistry: A unified and materialsgeneral strategy for the template-based synthesis of nanocrystalline solids. J. Solid State Chem. 2008, 181, 1509-1523.

[37] Wang, J. L.; Yang, Q.; Zhang, Z. D.; Sun, S. Phasecontrolled synthesis of transition metal phosphide nanowires via new Ullmann-type reactions. Chem. Eur. J. 2010, accepted.

[38] Wang, J. L.; Yang, Q.; Zhang, Z. D. Selective synthesis of magnetic $\mathrm{Fe}_{2} \mathrm{P} / \mathrm{C}$ and $\mathrm{FeP} / \mathrm{C}$ core/shell nanocables. J. Phys. Chem. Lett. 2010, 1, 102-106.

[39] Kumar, S.; Nann, T. Shape control of II-VI semiconductor nanomaterials. Small 2006, 2, 316-329.

[40] Robb, D. T.; Privman, V. Model of nanocrystal formation in solution by burst nucleation and diffusional growth. Langmuir 2008, 24, 26-35.

[41] Predel, B. Fe-P (Iron-Phosphorus). Madelung, O., Ed.; SpringerMaterials-The Landolt-Börnstein database. http:// www.springermaterials.com, DOI: 10.1007/10474837_1325.

[42] Fujii, H.; Uwatoko, Y.; Motoya, K.; Ito, Y.; Okamoto, T. Neutron scattering investigation of itinerant electron system $\mathrm{Fe}_{2}$ P. J. Phys. Soc. Jpn. 1988, 57, 2143-2153. 